A febrile seizure has long been recognized as the most common complication of exanthem subitum, occurring in 22 percent of 581 patients reported in 11 publications (Millichap JG. Febrile Convulsions. New York, Macmillan, 1968). Evidence for an encephalitic process and a direct involvement of the brain was lacking, and the degree of fever was considered sufficient to explain the frequent complication of convulsions. The present report demonstrates that seizures associated with exanthem subitum and fever are not always simple in type. They are occasionally prolonged and complex and a manifestation of encephalitis or encephalopathy.

\title{
E COLI HEMORRHAGIC COLITIS AND NEUROLOGIC SYMPTOMS
}

The neurological manifestations of hemorrhagic colitis in seven kindergarten children with Escherichia coli 0157:H7 infection are reported from the Saitama Children's Medical Center, Saitama, Japan. Generalized convulsions, occurring in 5 comatose patients, were intractable in 3 and required toxic doses of anticonvulsants for their control. Two patients died after 5 and 7 days of illness. Transient symptoms, delayed in onset for 2 to 6 weeks and after consciousness improved, included phrenic nerve paralysis in 1 patient, neurogenic urinary incontinence in 3 , gaze nystagmus in 3 , action tremor and oral dyskinesia in 1, and vertigo in 1 patient. Pyrexia and hemolytic uremic syndrome were seen only in patients with neurologic complications. Diarrhea, bloody diarrhea, and abdominal pain were more frequent and severe in this group compared to 7 patients with no neurologic symptoms who were admitted to hospital. Contaminated well water was the source of infection, and verotoxin-I (Shiga-like toxin I) was the likely cause of neurological symptoms. (Hamano $S$ et al. Neurological manifestations of hemorrhagic colitis in the outbreak of Escherichia coli 0157:H7 infection in Japan. Acta Paediatr May 1993; $\underline{82:}$ 454-458). (Respond: S Hamano MD, Division of Neurology, S Children's Medical Center, 2100 Magome, Iwatsuki, Saitama 339, Japan).

COMMENT. Fever, high white blood cell count, young or old age, and prolonged antimotility drug use are risk factors for complications of this strain of E. coli infection. The neurologic and other complications of foodborne and waterborne infections, pollutants, and additives, are covered in Environmental Poisons in Our Food, Chicago, PNB Publishers.

\section{SEVERE REACTIONS TO DTP VACCINE}

Severe reactions experienced by 60 children within 48 hours of DTP immunization were reported from the UCLA School of Medicine, Los Angeles, CA. These included seizures in 40 or $66 \%$ ( 6 with fever $>40.5^{\circ} \mathrm{C}$ ), collapse episodes in 14, and persistent crying in 6 . Subjects with encephalopathy were not included in the study. Those with seizures had a high rate of personal and 